\title{
Kinematic distances for southern pulsars: Gum \& Carina
}

\author{
Bärbel Koribalski \\ ATNF, CSIRO, Australia \\ Simon Johnston \\ RCfTA, University of Sydney, Australia \\ Joel Weisberg \\ Carleton College, MN, USA
}

\section{Introduction: $\mathrm{H}_{\mathrm{I}}$ absorption measurements towards pulsars}

We have observed 21-cm absorption and emission spectra in the direction of about 20 southern pulsars with the Parkes telescope to derive their kinematic distances and to study the interstellar medium. In each case we thoroughly investigated the environment of each pulsar as well as sources along the line of sight which may contribute to the pulsars' dispersion measure (DM).

Some of the results, which are published in Koribalski et al. (1995) and Johnston et al. (1996), are included in the compilation of $\mathrm{H} \mathrm{I}$ absorption measurements by Joel Weisberg in this volume. In the following we present a detailed discussion on pulsars toward 1) the Gum Nebula and 2) the Carina Arm.

\section{The Gum Nebula: Model vs Observations}

It has long been recognised that pulsars near $l \sim 260^{\circ}$ appear to have anomalously high DMs. This was first apparent for the Vela pulsar which has a measured DM of $79 \mathrm{~cm}^{-3} \mathrm{pc}$, whereas its 'expected' DM at a distance of $500 \mathrm{pc}$ would be only $\sim 10 \mathrm{~cm}^{-3} \mathrm{pc}$. This, and other excess DMs can be attributed to a large, diffuse ionized region known as the Gum Nebula.

The origin of the Gum Nebula is not entirely clear: it may be a) the fossil Strömgren sphere from either the relatively recent Vela supernova explosion or a 1-2 Myr old supernova explosion, b) a remnant with ionization currently maintained by the stars $\gamma^{2}$ Vel and $\zeta$ Pup, or c) a normal $\mathrm{H}$ il region surrounding those stars.

For our purposes, we note that a sphere with parameters resembling those of the Gum Nebula is explicitly built into the Taylor \& Cordes (1993) model of the Galactic electron density distribution. The sphere is centred at $(l, b)=\left(260^{\circ}, 0^{\circ}\right)$, slightly offset from the Vela pulsar, and located at a distance of $500 \mathrm{pc}$. Its electron density is constant over a radius of $130 \mathrm{pc}, n_{\mathrm{e}}=0.25 \mathrm{~cm}^{-3}$, and then follows a Gaussian distribution with a scale length of $50 \mathrm{pc}$.

There are now 7 pulsars which have independent distance measurements and lie in or behind the Gum Nebula (PSRs J0738-4042, J0742-2822, J0835-4510, 
J0837-4135, J0908-4913, J0942-5552 and J1001-5507). The distances derived using the Taylor \& Cordes model are consistent with those obtained kinematically for all these pulsars except for PSR J0742-2822 which lies marginally below the kinematically derived lower limit. For PSR J0738-4042, however, we strongly favour a distance close to the $\mathrm{H}$ I distance limit of $2 \mathrm{kpc}$ rather than the $11 \mathrm{kpc}$ claimed by the Taylor \& Cordes model. We argue that a density enhancement in the Gum Nebula or possibly a large, nearby H II region (e.g. BBW 56) contributes to the DM. Our final conclusion is that the relatively simple Taylor $\&$ Cordes model matches well with the data.

\section{The Carina Arm: a laboratory for streaming motions}

The Carina region of the southern Milky Way has long been a rich area of study. Bok \& Lindsay (1937) proposed that a spiral arm runs tangentially to the line of sight in this direction near $l=280^{\circ}$. Further evidence came from a comparison of radio and $\mathrm{H} \alpha$ observations both of which showed an abrupt cut-off in intensity at longitudes just below $280^{\circ}$. A study of $\mathrm{OB}$ stars and $\mathrm{H}$ II regions in this area led to similar conclusions. The generally established picture is that the Carina Arm builds a tangent to the solar circle at $l=283^{\circ}$ and opens outward at 2 $\mathrm{kpc}$ from the Sun towards $l=295^{\circ}$. The Arm then extends out to $20 \mathrm{kpc}$ at increasingly higher longitudes. The streaming motions imposed by the Carina Arm result in spectra with a much larger velocity range than expected from models of the Galactic rotation curve. The excess velocities are usually referred to as 'forbidden' velocities. To determine pulsar distances in this region we have studied their environment, especially nearby gas clouds for which independent velocity and distance measurements are available (e.g. Brand \& Blitz 1993). That way, upper and lower kinematic distance limits were derived for 4 pulsars in the direction of the Carina Arm (PSRs J1048-5832, J1056-6258, J1157-6224, and J1224-6407). In accord with Brand \& Blitz and others, we show that the rotation curve model of Fich et al. (1989) does not agree well with the $\mathrm{HI}$ emission velocities in the Carina spiral arm. We have conservatively assigned the lower distance limit as the tangent point with a large uncertainty to PSRs J1157-6224 and J1224-6407. Although distances to nearby objects such as H II regions are useful, care must be taken in interpreting a smoothed velocity field obtained from these data as they are sparse at distances beyond a few kpc.

\section{References}

Bok, B.J., Lindsay, E.M. 1937, Ann. Harvard College Obs., 105, 327

Brand, J., Blitz, L. 1993, A\&A, 275, 67

Fich, M., Blitz, L., Stark, A.A. 1989, ApJ, 342, 272

Johnston, S., Koribalski, B., Weisberg, J.M., Wilson, W. 1996, MNRAS, 279, 661

Koribalski, B., Johnston, S., Weisberg, J.M., Wilson, W. 1995, ApJ, 441, 756

Taylor, J.H., Cordes, J.M. 1993, ApJ, 411, 674 